\title{
Field Mapping Without Reference Scan Using Asymmetric Echo-Planar Techniques
}

\author{
Rolf Gruetter* and Ivan Tkáč
}

\begin{abstract}
Improvements in $\mathrm{B}_{0}$ mapping and shimming were achieved by measuring the static field information in multiple subsequent echoes generated by an asymmetric echo-planar readout gradient train. With careful compensation, eddy current effects were shown to affect the adjustment of the shim coils minimally. In addition to reducing the time required for field mapping by two-fold, the sensitivity was simultaneously optimized irrespective of the prevalent $T_{2}^{*}$ present, thereby minimizing the error of the static field measurement to below $0.1 \mathrm{~Hz}$. With adiabatic low flip-angle excitation, the time required for field mapping was below 1 second. Magn Reson Med 43:319-323, 2000. (c) 2000 Wiley-Liss, Inc.
\end{abstract}

Key words: shimming; EPI; field mapping; $B_{0}$ imaging; spectroscopy; BOLD

In vivo, optimal spectral resolution is difficult to achieve due to sample- and tissue- dependent susceptibility effects. Although the latter can be regarded as microscopic susceptibility effects, which are extremely difficult to eliminate, the former is caused mainly by the air-tissue or bone-tissue interface and depends on geometry and orientation. Adjusting shim coil correction currents can reduce macroscopic susceptibility effects. Many shim methods have been described (see Refs. 1 and 2 and references therein). Most have used linear shim coils $(X, Y, Z)$, while some have used higher order shim coils (3). The adjustment of second-order shimming using FASTMAP has been described in the past years $(1,2,4,5)$. This method is based on a sparse, yet efficient sampling of the magnetic field along a limited number of projections, which effectively reduces the time required to obtain the static field information and to extract the required correction current changes for the shim coils. Traditionally, the static field information has been encoded by adding an extra delay to the sequence, as in many similar MRI- or EPI-based approaches (6). However, two limitations can be stated for all these approaches, namely (a) the requirement to acquire an additional reference scan to compensate for sequence-dependent eddy current effects and (b) that the choice of $\tau$ must be optimized because of the variable inhomogeneity of the static field to minimize the error in shim coil adjustment, as described in Eq. [7] in Ref. 1. When starting the shimming process, it is often observed that at least one of the linear shims requires substantially more adjustment. In this case, the choice of $\tau$ cannot be optimal for all readout gradient orientations due to in-slice dephasing effects. The

Department of Radiology, University of Minnesota, Minneapolis, Minnesota. Grant sponsor: National Institutes of Health; Grant number: RR08079.

${ }^{*}$ Correspondence to: Rolf Gruetter, Center for MR Research, 2021 6th Street SE, Minneapolis, MN 55455. E-mail: gruetter@cmrr.umn.edu

Received 9 July 1999; revised 6 October 1999; accepted 22 October 1999.

(c) 2000 Wiley-Liss, Inc. advantage of encoding the field with an extra delay $\tau$ preceding any switched gradients was that the static field was measured without any distortions associated with gradient switching, i.e., eddy currents. However, a recent report suggested that alternating the readout gradient of an MRI sequence may provide enough accuracy to extract the field information from a comparison of the phase of the two subsequently generated echoes (7). Unfortunately, such an approach is in principle highly susceptible to eddy current effects. Based on the large variation in $\mathrm{B}_{0}$ measurements of $0.05 \mathrm{ppm}(3 \mathrm{~Hz})$ reported, it remained unclear to what extent residual eddy current effects permit collection of field maps suitable for in vivo spectroscopy and echo-planar imaging. In an attempt to further optimize the performance and speed of the aforementioned FASTMAP and any field mapping MRI method based on phase difference measurements, we implemented multiple gradient-echo readouts using an approach based on asymmetric echo-planar readout gradient trains (8-10). Experience showed that distortions of the field measurement due to eddy current effects were minor even for the demands imposed by in vitro spectroscopy of phantoms. This paper describes the implementation of this new, self-referencing scheme, for which the acronym fast, automatic shim technique using echo-planar signal readout for mapping along projections, FAST(EST)MAP, is proposed. The proposed scheme can also be incorporated into traditional MRIbased phase difference methods for mapping the magnetic field, such as in Refs. 3, 7, 11-13.

\section{MATERIALS AND METHODS}

All experiments were carried out on a 4 T/90-cm magnet (Oxford Magnet Technologies, Oxford, UK) equipped with a standard whole-body gradient coil (AS25, Siemens AG, Erlangen, Germany, $25 \mathrm{mT} / \mathrm{m}$ in $600 \mu \mathrm{s}$ ) as well as a $38-\mathrm{cm}$ head gradient coil (Magnex Ltd. Abingdon, UK, $32.5 \mathrm{mT} / \mathrm{m}$ in $200 \mu \mathrm{s})$. The system was interfaced to a Varian INOVA console and all shimming software was written in C programming language and implemented in menu-driven format that minimized operator interaction and ensured operator-independent performance.

Eddy currents were carefully minimized using a quantitative method to map and compensate eddy current fields (14).

In vivo performance of the shim method was tested on human subjects at $4 \mathrm{~T}$ after they gave informed consent according to procedures and forms approved by the Institutional Review Board. Localization of volumes of interest was based on anatomic MRI using multi-slice RARE (TR = $3 \mathrm{sec}, \mathrm{TE}=60 \mathrm{msec}, 8$ echoes recorded per excitation) (15). RF power absorption was verified to be within FDA guidelines. 


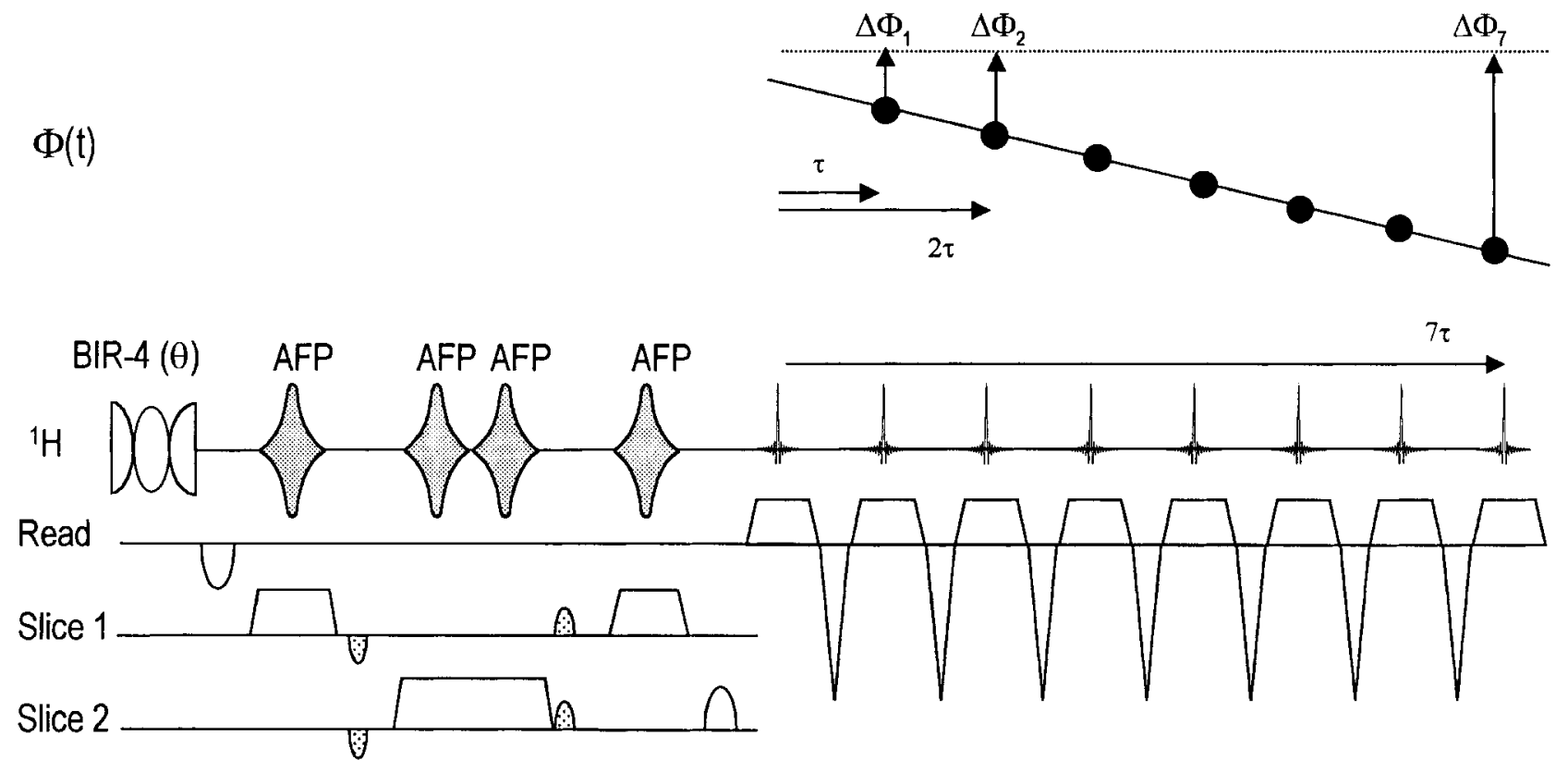

FIG. 1. Implementation of echo-planar methods into FASTMAP. From the $N$ echoes recorded ( $N=8$ in this example), $N-1$ phase maps are extracted $\left(\Delta \phi_{n}\right)$ and analyzed and the resulting field information is calculated from $\Delta \phi_{n} / n \tau$ for each of the $N-1$ projections using a weighted $\chi^{2}$ minimization (Eq. [1]), thereby ensuring optimal accuracy in minimal experimental time. Shaded gradient lobes indicate spoiler gradients used to minimize coherence generation when using very short TR.

For shimming and comparison, we used the fully adiabatic FASTMAP pulse sequence, exactly as described recently in Fig. 1 of Ref. 4. The method relies on measuring the phase difference of projections acquired with an extra delay $\tau$ in the first echo period to those acquired without such a delay. The resulting $B_{0}$ field is thus encoded without any confounding effects from eddy currents when using sufficiently long repetition times TR, which was verified by comparing results obtained at different TR. Measurements obtained with the sequence of Fig. 1 in Ref. 4 served as a reference for comparison as follows: In phantoms, we initially shimmed a localized volume using FASTMAP, such that residual shim terms were below 0.1 $\mathrm{Hz} / \mathrm{cm}$ for the linear coils and below $0.1 \mathrm{~Hz} / \mathrm{cm}^{2}$ for the second-order terms, which was verified to result in a narrow line width of metabolites in aqueous solutions (below $1 \mathrm{~Hz}$ for 27-ml volumes). Then the sequence based on an echo-planar readout gradient train was applied and the resulting differences in calculated polynomial coefficients were attributed to eddy current effects.

\section{RESULTS AND DISCUSSION}

The sequence used in this implementation (Fig. 1) is similar to the fully adiabatic double-spin echo described recently (4), with the following modifications: First, the previously used adiabatic half-passage pulse generating a nominal $90^{\circ}$ excitation was replaced with a plane-rotation adiabatic BIR-4 pulse (16) of 3-msec duration. The phase of the middle segment determines the flip angle, which was set to below $90^{\circ}$ (typically $\theta=45^{\circ}$ ). A spatially uniform $45^{\circ}$ flip angle is the optimal Ernst angle for TR/T $\sim 0.3$ (17). A TR of $0.3 \mathrm{sec}$ is thus close to the optimum, given that $\mathrm{T}_{1}$ of brain water is on the order of 1 sec. Gaussian apodization of the echoes was used for smoothing the projections. Using a flip angle below $90^{\circ}$ allowed to decrease the repetition time to $0.1 \mathrm{sec}$. A TR of $0.1 \mathrm{sec}$ implies that the field information can be mapped in 0.6 sec using FASTMAP. However, this was achieved at the expense of sensitivity and thus accuracy of the shim current adjustments was reduced (as pointed out previously in Ref. 1). Therefore, we chose a typical TR of $0.3 \mathrm{sec}$, which maintains a minimal time required for the field mapping process of less than 2 sec. Such a short measuring time is insignificant compared to other adjustment procedures required for in vivo imaging and spectroscopy.

The second major change made to the adiabatic version of FASTMAP (4) consisted of the replacement of the initial static field encoding delay $\tau$ (inserted previously on alternating scans immediately following the excitation pulse) with an echo-planar readout train (Fig. 1).

Gradient magnitudes and rise times were carefully selected below the specified maximum performance to minimize eddy current distortions: Rise times were typically between 0.2 and $0.6 \mathrm{msec}$. The readout gradient strengths were on the order of $6 \mathrm{mT} / \mathrm{m}$, which resulted in a FOV of $25 \mathrm{~cm}$ using a $50 \mathrm{kHz}$ receiver bandwidth. During negative readout gradient lobes ("odd echoes") the signal was not acquired, instead, a triangular gradient shape was used to minimize eddy current effects and to minimize inter-echo spacing. The minimal inter-echo time $\tau$ was limited by the acquisition time and the duration of the rewinding triangular gradient (Fig. 1), which in turn was limited by the maximum gradient strength and the risetime of the gradient system. Eddy current effects were expected to mainly affect the linear terms, because second-order eddy current terms are generally negligible when using actively shielded gradient coils. 
FIG. 2. ${ }^{1} \mathrm{H}$ MR projections collected from a bar selected along $x$ at multiple echo times $n \tau(\tau=10 \mathrm{msec}, n=1 \ldots$ 10) measured up to $T E=100 \mathrm{msec}$. Differences in tissue $T_{2}^{*}$ were due to cerebrospinal fluid spaces associated with the occipital horns (arrows).

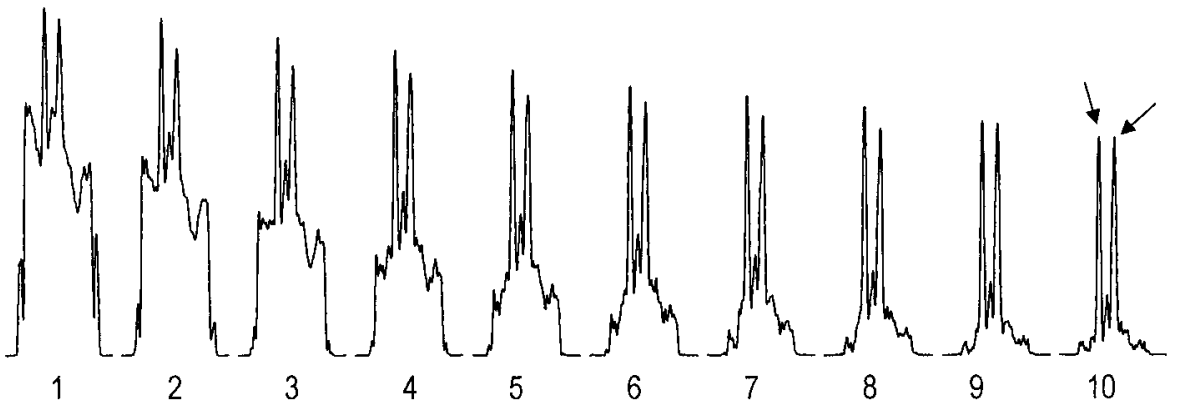

With an acquisition time of $2.7 \mathrm{msec}$, an interval spacing of $\tau=5.5$ msec was readily achieved with the whole-body gradient coil, where the rise time was $600 \mu \mathrm{s}$. After Gaussian apodization, the $N$ echoes acquired per orientation (with a field-encoding delay of $(n-1) \tau$ ) were Fourier transformed and the resulting $N$ complex projections were stored to disk. Field information was extracted from these projections by a point-by-point calculation of the phase difference between the $n$-th echo and the first echo $(n>1)$ using methods for one-dimensional phase unwrapping described previously (1) and by dividing the phase difference by $(n-1) \tau$. Polynomials were fitted to all $N-1$ phase function along the specific bar and the resulting polynomial coefficients $\mathrm{a}_{n}$ were calculated and averaged using a weighted $\chi^{2}$-minimization, based on the respective rootmean-square deviation (RMSD) $\sigma_{n}$ using the equation

$$
A=\sum_{n=1}^{N-1} a_{n} \sigma_{n}^{-2}\left[\sum_{n=1}^{N-1} \sigma_{n}^{-2}\right]^{-1}
$$

This type of weighted averaging automatically ensured that when the encoding delay $n \tau$ was large and the magnetization reduced due to $\mathrm{T}_{2}^{*}$-dephasing, which includes both macroscopic field inhomogeneities (caused by, e.g., an unadjusted initial shim setting) and intrinsic tissue $\mathrm{T}_{2}^{*}$, the RMSD of the fit to the phase map was increased and, therefore, the corresponding polynomial coefficient contributed less to the average.

The theoretical error in the field determination (Eq. [7] in Ref. 1) is rewritten here with the factors dependent on $\mathrm{T}_{2}^{*}$ and $\tau$ as

$$
\sigma_{p p m}^{-2}\left(n \tau, T_{2}^{*}\right)=\frac{[n \tau]^{2}}{1+e^{2 n \tau / T 2^{*}}}
$$

Calculating from Eq. [2], the average error due to the $N$ measurements made at $n \tau(n=1 . . \mathrm{N})$ indicated that for a range of $\mathrm{T}_{2}^{*}$ from $0.005 \mathrm{sec}$ to $0.3 \mathrm{sec}$ the error is always less than that achievable by a single encoding step with a delay $N \tau$. When integrating over all $(N-1)$ phase measurements we calculated that the error rapidly decreased with $n \tau$, reaching close to optimal values for $n \tau>0.02 \mathrm{sec}$. In addition, the simulations showed a distinct improvement in relative error when $T_{2}^{*}$ was larger than the echo spacing $\tau$ (not shown).

Figure 2 shows the Fourier transformed projection along $x$ (from right to left) acquired from a well-shimmed human brain at $4 \mathrm{~T}$, indicating that sufficient sensitivity was present for all 10 echoes, which spanned a TE of 10-100 msec. When mapping the field under poorly shimmed conditions, in-slice dephasing results in a reduction of the apparent $\mathrm{T}_{2}^{*}$ and the measurements using the later echoes will not be reliable and result in an increased RMSD $\sigma_{n}$ of the polynomial fit. A recent modification of FASTMAP suggested to use a weighted fitting function to account for reduced sample sensitivity, e.g., when measuring across signal voids such as bone or areas with very short $\mathrm{T}_{2}^{*}(2)$. We have incorporated such an approach, however, to avoid increased weighting towards the long $\mathrm{T}_{2}^{*}$ signals, such as water in cerebrospinal fluid or cysts at longer encoding delays $n \tau$, as indicated by the arrows in Fig. 2, a modified weighted fitting was implemented, as follows: All points with a signal-to-rms-noise ratio above a predefined threshold (typically 10) were equally weighted $(\sigma=1)$ and all those below this threshold were assigned the weight $\sigma=0$.

The self-referencing sequence was tested by comparison with the fully adiabatic FASTMAP sequence (4) using the time delay $\tau=15 \mathrm{msec}$ inserted into the first echo period prior to any gradient switching. With an inter-echo spacing of $5.5 \mathrm{msec}$ and a 38-cm actively shielded head gradient insert (Magnex, Abingdon, UK), we observed a maximal deviation in first-order polynomial coefficients of 0.42 $\mathrm{Hz} / \mathrm{cm}$ and at most $0.06 \mathrm{~Hz} / \mathrm{cm}^{2}$ for the second-order coefficients. These deviations translated into a maximum deviation for the calculated shim coefficients of 0.42 $\mathrm{Hz} / \mathrm{cm}$ and $0.06 \mathrm{~Hz} / \mathrm{cm}^{2}$, respectively. For a standard body gradient coil (AS25, Siemens, Germany), we observed maximum deviations of $0.37 \mathrm{~Hz} / \mathrm{cm}$ and $0.05 \mathrm{~Hz} / \mathrm{cm}^{2}$, respectively. The deviation was further minimized by using the six projections oriented along the six diagonals of a cube, but intersecting at the center-of-mass (i.e., $x y, y x$, $x z, z x, y z, z y)$, as described in Ref. 1. Maximal deviations of $0.13 \mathrm{~Hz} / \mathrm{cm}$ and $0.07 \mathrm{~Hz} / \mathrm{cm}^{2}$ in shim coefficients were measured between the two procedures for field mapping when using the head gradient coil. For the body gradient coil, we observed maximum deviations of $0.35 \mathrm{~Hz} / \mathrm{cm}$ and $0.04 \mathrm{~Hz} / \mathrm{cm}^{2}$. In an aqueous phantom containing $250 \mathrm{mM}$ glucose, this resulted in a $0.1-\mathrm{Hz}$ broader line width for a 27-ml volume when using the echo-planar based scheme using either gradient coil, an error that is well-tolerable when considering that in vivo line widths are typically on the order of several Hertz. At 4 T, excellent field homoge- 


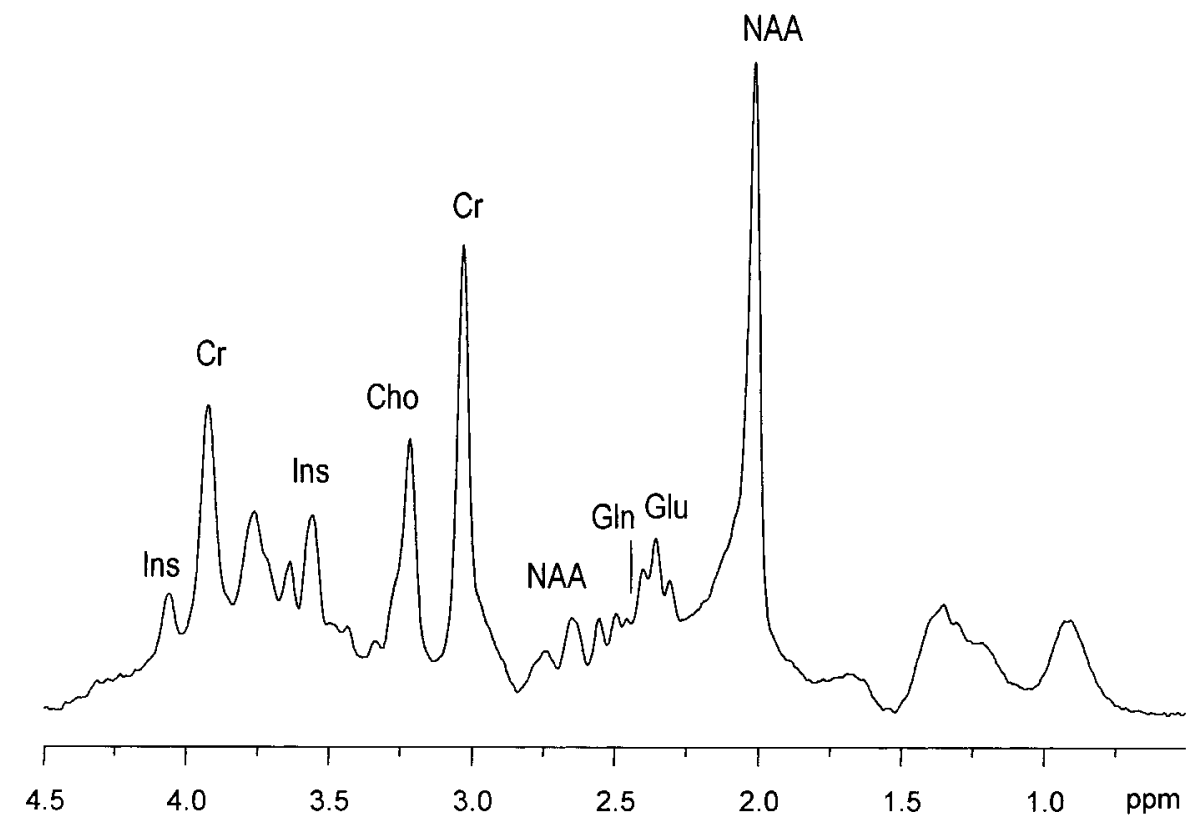

FIG. 3. In vivo ${ }^{1} \mathrm{H}$ NMR spectrum from the human occipital lobe using a recently described implementation of STEAM (18), adapted to the human 4-T system. Echo time TE was $20 \mathrm{msec}$, TR $=4 \mathrm{sec}$, and $\mathrm{TM}=40 \mathrm{msec}$. Spectra were from 27-ml volumes in the human occipital lobe and are shown with a mild Gaussian apodization that did not reduce the line width.

neity was achieved using the new scheme, as illustrated with a ${ }^{1} \mathrm{H}$ spectrum of the human brain in Fig. 3. Typically, before shimming the line width of water in gray matter was on the order of $30 \mathrm{~Hz}$ at $4 \mathrm{~T}\left(27 \mathrm{~cm}^{3}\right)$ and $100 \mathrm{~Hz}$ at $9.4 \mathrm{~T}$ (64 $\mu \mathrm{l}$ ). The proposed shim technique resulted in line widths that were validated to be the same when reducing the voxel size to $1 \mathrm{~cm}^{3}$ and $8 \mu \mathrm{l}$, respectively, from which we concluded that (macroscopic) contributions to $\mathrm{T}_{2}^{*}$ (line width) were minimal for such volume sizes.

We conclude that with a reasonable effort in eddy current compensation, their effect can be minimized in the proposed scheme to a level that is insignificant for most in vivo spectroscopy applications and certainly for all imaging applications, including EPI. Eddy current effects can be minimized by decreasing the readout gradient strength and slew rate, which requires an increase in the echo spacing $\tau$, which may not be desirable when attempting to minimize $\mathrm{T}_{2}$ losses. Alternatively, because the most relevant eddy current distortions for the proposed mapping are the linear terms, it is possible to calibrate the triangular rewinding gradient lobes against, e.g., measurements using an eddy current-insensitive procedure as a reference (using, e.g., the pulse sequence in Fig. 1 of Ref. 4) to provide field maps that are independent of the echo number $n$. This procedure may also be required on systems with slight imbalances between positive and negative gradients strengths. The proposed scheme of Fig. 1 has been tested successfully on two additional systems: A 7-T /90-cm magnet with a 38-cm actively shielded head gradient coil and a 9.4 T/31-cm magnet with an 11-cm actively shielded gradient insert. In all cases, we found eddy currents to produce minimal distortions in the measured $\mathrm{B}_{0}$ field, suggesting that the proposed scheme will perform adequately on most contemporary systems.

For MRI-based field mapping, the minimal field information for shimming all first-and second-order shim coils requires that at least three image planes are acquired. A separate reference scan is needed, resulting in at least 6 excitations when using very fast single-shot imaging sequences such as EPI (6). The echo-planar imaging approach thus has mapping times comparable to the sequence proposed herein, but requires corrections due to image distortions acquired mainly in the phase encode direction, longer processing times and the use of an encoding delay $\tau$. Other $B_{0}$ field imaging methods (12) require far more excitations, resulting in 5 min required for shimming (3). Including data acquisition, calculation of shim correction currents and download to the shim computer, the proposed FASTMAP-based approach required less than $15 \mathrm{sec}$, which is a substantial improvement. This suggests that a more efficient reduction of field mapping time and computational effort, and improved reliability was achieved than possible with any of the other current techniques.

Nevertheless, it should be emphasized that the proposed approach based on an acquisition of the field information without acquiring an extra reference scan can be extended to MRI-based field mapping techniques, provided the phase encoding for the second spatial axis is acquired with sequential excitations, such as during a FLASH-type imaging scheme.

We conclude that with the proposed scheme the time required to measure the static field $B_{0}$ can be minimized to a level that eliminates shimming as an obstacle for the routine use of clinical MR spectroscopy.

\section{REFERENCES}

1. Gruetter R. Automatic, localized in vivo adjustment of all first- and second-order shim coils. Magn Reson Med 1993;29:804-811.

2. Shen J, Rycyna RE, Rothman DL. improvements on an in vivo automatic shimming method (FASTERMAP). Magn Reson Med 1997;38: 834-839.

3. Spielman DM, Adalsteinsson E, Lim KO. Quantitative assessment of improved homogeneity using higher-order shims for spectroscopic imaging of the brain. Magn Reson Med 1998;40:376-382. 
4. Gruetter R, Weisdorf SA, Rajanayagan V, Terpstra M, Merkle H, Truwit CL, Garwood M, Nyberg SL, Ugurbil K. Resolution improvements in in vivo 'b9H NMR spectra with increased magnetic field strength. J Magn Reson 1998;135:260-264.

5. Gruetter R, Boesch C. Fast, non-iterative shimming on spatially localized signals: in vivo analysis of the magnetic field along axes. J Magn Reson 1992;96:323-334.

6. Reese TG, Davis TL, Weisskoff RM. Automated shimming at $1.5 \mathrm{~T}$ using echo-planar image frequency maps. J Magn Reson Imag 1995;5:739745 .

7. Kanayama S, Kuhara S, Satoh K. In vivo rapid magnetic field measurement and shimming using single scan differential phase mapping. Magn Reson Med 1996;36:637-642.

8. Hennel F, Nedelec JF. Interleaved asymmetric echo-planar imaging. Magn Reson Med 1995;34:520-524.

9. Feinberg DA, Turner R, Jakab PD, von Kienlin M. Echo-planar imaging with asymmetric gradient modulation and inner-volume excitation. Magn Reson Med 1990;13:162-169.

10. Yang QX, Posse S, Lebihan D, Smith MB. Double-sampled echo-planar imaging at 3 Tesla. J Magn Reson B 1996;113:145-150.
11. Schneider E, Glover G. Rapid in vivo proton shimming. Magn Reson Med 1991;18:335-347.

12. Webb P, Macovski A. Rapid, fully automatic, arbitrary-volume in vivo shimming. Magn Reson Med 1991;20:113-122.

13. Wen H, Jaffer FA. An in vivo automated shimming method taking into account shim current constraints. Magn Reson Med 1995;34:898-904.

14. Terpstra M, Andersen P, Gruetter R. Localized eddy current compensation using quantitative field mapping. J Magn Reson 1998;131:139143.

15. Hennig J, Nauerth A, Friedburg H. RARE imaging: a fast imaging method for clinical MR. Magn Reson Med 1986;3:823-833.

16. Garwood M, Ke Y. Symmetric pulses to induce arbitrary flip angles with compensation for RF inhomogeneity and resonance offsets. J Magn Reson 1991;94:511-525.

17. Ernst RR, Bodenhausen G, Wokaun A. Principles of nuclear magnetic resonance in one and two dimensions. The International series of monographs on chemistry; 14. Oxford: Oxford University Press; 1987. p 156.

18. Tkac I, Starcuk Z, Choi I-Y, Gruetter R. In vivo 1 H NMR spectroscopy of rat brain at $1 \mathrm{~ms}$ echo time. Magn Reson Med 1999;41:649-656. 